\title{
Methadone Medical Maintenance in Primary Care
}

\section{An Implementation Evaluation}

Joseph O. Merrill, MD, MPH, ${ }^{1,6}$ T. Ron Jackson, MSW, ${ }^{2}$ Beryl A. Schulman, PhD, MSW, ${ }^{1,6}$

Andrew J. Saxon, MD, ${ }^{3}$ Asaad Awan, Pharm D, ${ }^{4}$ Sonja Kapitan, Pharm D, MPH,

Molly Carney, PhD, ${ }^{6}$ Lyndia C. Brumback, PhD, ${ }^{1,5,7}$ Dennis Donovan, PhD ${ }^{3,6}$

${ }^{1}$ Departments of Medicine, ${ }^{2}$ Social Work, ${ }^{3}$ Psychiatry and Behavioral Sciences, ${ }^{4}$ Pharmacy, and ${ }^{5}$ Biostatistics, ${ }^{6}$ Alcohol and Drug Abuse

Institute, and ${ }^{7}$ Center for AIDS Research, University of Washington, Seattle, WA, USA.

\begin{abstract}
BACKGROUND: Methadone is effective treatment for opioid addiction, but regulations restrict its use. Methadone medical maintenance treats stabilized methadone patients in a medical setting, but only experimental programs have been studied.
\end{abstract}

OBJECTIVE: To evaluate the implementation of the first methadone medical maintenance program established outside a reseach setting.

DESIGN: One-year program evaluation.

SETTING: A public hospital and a community opioid treatment program.

PARTICIPANTS: Methadone patients with > 1 year of clinical stability. Eleven generalist physicians and 4 hospital pharmacists.

INTERVENTIONS: Regulatory exemptions were requested. Physicians and pharmacists were trained. Patients were transferred to the medical setting and permitted 1-month supplies of methadone.

MEASUREMENTS: Patient eligibility and willingness to enroll, treatment retention, urine toxicology results, change in addiction severity and functional status, medical services provided, patient and physician satisfaction, and physician attitudes toward methadone maintenance.

RESULTS: Regulatory exemptions were obtained after a 14-month process, and the program was cited in federal policy as acceptable for widespread implementation. Forty-nine of 684 patients (7.2\%) met stability criteria, and 30 enrolled. Twenty-eight were retained for 1 year, and 2 transferred to other programs. Two patients had opioid-positive urine tests and were managed in the medical setting. Previously unmet medical needs were addressed, and the Addiction Severity Index (ASI) medical composite score improved over time $(P=.02)$. Patient and physician satisfaction were high, and physician attitudes toward methadone maintenance treatment became more positive $(P=.007)$.

CONCLUSIONS: Methadone medical maintenance is complex to arrange but feasible outside a research setting, and can result in good clinical outcomes.

KEY WORDS: methadone; heroin addiction; opioid-related disorders; outcome and process assessment.

DOI: $10.1111 /$ j.1525-1497.2005.04028.x

J GEN INTERN MED 2005; 20:344-349.

$\mathrm{O}$ pioid addiction is a chronic medical condition most effectively treated with medication and counseling. ${ }^{1}$ Longterm methadone treatment reduces the individual and societal costs of addiction, ${ }^{2,3}$ and is more effective than methadone treatment of limited duration. ${ }^{4-6}$ Access to methadone treat-

Accepted for publication October 22, 2004

None of the authors has any conflicts of interest related to this manuscript.An earlier version of this work was presented at the Society of General Internal Medicine annual meeting, Atlanta, GA, May 1, 2002.

Address correspondence and requests for reprints to Dr. Merrill: Harborview Medical Center, 325 Ninth Avenue, Box 359780, Seattle, WA 98104 (e-mail: joem@u.washington.edu). ment in the United States is restricted to opioid treatment programs (OTPs), which are isolated from medical practice and highly regulated. ${ }^{7}$ This has led to inadequate funding, inconsistent access to care, large treatment programs that face community opposition to expansion, and a medical system that treats the complications of addiction rather than the primary problem. ${ }^{8}$

Treatment of methadone maintenance patients by physicians outside the existing OTP system has been attempted using a model known as methadone medical maintenance. ${ }^{9,10}$ Successful long-term methadone maintenance patients transfer from traditional programs to a medical setting and are allowed fewer treatment visits and more take-home medication. The medical setting can destigmatize treatment, reduce contacts with unstable patients, and facilitate treatment of neglected medical problems. ${ }^{11}$ Previous long-term observational studies of methadone medical maintenance documented good treatment retention and few safety problems. ${ }^{12,13}$ Short-term randomized trials comparing methadone medical maintenance with regular clinic-based methadone treatment have demonstrated similar addiction-related outcomes and improved patient satisfaction. ${ }^{14,15}$ These results are based on experimental programs authorized through the Investigational New Drug (IND) process of the U.S. Food and Drug Administration (FDA). $9,10,12-15$

Our goal was to integrate methadone medical maintenance into the continuum of care for opioid dependence by seeking nonexperimental regulatory approval for a program based in a general internal medicine clinic. Our program evaluation sought to determine the proportion of patients eligible and willing to enter methadone medical maintenance. We also tested whether these patients would remain stable, as measured by their retention in treatment, urine toxicology results, and change in addiction severity and functional status over time. Other measures included the utilization of primary care medical services, patient and physician satisfaction, and change in physician attitudes toward methadone treatment after training and methadone medical maintenance practice.

\section{METHODS}

\section{Policy Development}

We sought to develop a methadone medical maintenance program that could be approved by all regulatory stakeholders outside the experimental (IND) process. This required a collaborative process of policy and clinical protocol development involving federal, state, and local regulatory agencies as well as clinical participants from Harborview Medical Center (HMC)an urban public teaching hospital-and Evergreen Treatment Services (ETS)—a nonprofit community OTP. We requested 
federal and state exemptions from opioid addiction treatment regulations to initiate an HMC methadone medical maintenance program affiliated with ETS. Exemptions included extended take-home dose privileges, dispensing of solid-form methadone in multidose containers, decreased time in treatment before eligibility for maximum take-home doses, and the ability of HMC to order methadone directly. Protocols for pharmacist assessment and dispensing of methadone were submitted to the Washington State Board of Pharmacy.

Financing for methadone services at HMC was provided by patients' existing payment sources. ETS received approximately one third of patient fees for maintaining space for transfers and for providing counseling, billing, and program licensing services. HMC received the remainder of patients' methadone treatment fees, with two thirds of the HMC share funding pharmacy services and one third funding medical clinic services. In addition, HMC received reimbursement for primary care services through patients' medical insurance or HMC's low-income program for uninsured patients.

\section{Program Participants}

All methadone maintenance patients at ETS were screened. Eligible patients picked up methadone no more than 3 times weekly and fulfilled the following requirements: reliable attendance, monthly urinalysis negative for illicit drugs for 12 months, no clinical or breathalyzer evidence of current alcohol abuse or dependence, no outstanding legal issues or unpaid program fees, and no untreated major psychiatric illness documented by ETS staff. ETS clinical staff discussed qualifying patients to evaluate evidence of their clinical stability, including social support, employment, and education. Ten of the most clinically stable patients were transferred in January 2000 so that program logistics could be tested. Twenty additional patients transferred 2 to 6 months later.

All 11 attending physicians who worked more than 1 session per week in the HMC adult medicine clinic agreed to participate. The 4 participating pharmacists were from the HMC pharmacy, which serves patients who receive care from the HMC inpatient services and outpatient clinics. No physician or pharmacist had previous addiction medicine or methadone maintenance training or experience.

\section{Program Features}

The initial provider training consisted of two 3-hour group sessions. The first included an interview with an eligible patient and a discussion of addiction and methadone treatment, in particular, clinical monitoring, dealing with instability, dose adjustment, and program logistics (especially record keeping and confidentiality). The second session involved a visit to ETS for review of methadone maintenance practices and discussion with staff. Ongoing clinical support included distribution of literature and clinical consultation as requested. Providers discussed cases and program logistics at 2 additional evening meetings during the first year.

Patients transferred to HMC with once or twice weekly methadone pick-up and became eligible for once-per-month take-home status within 3 to 6 months. Patients picking up 3 times per week at ETS began twice weekly pick-up for 2 months before transfer. Dispensing visits occurred in the medical clinic before usual business hours; pharmacists assessed patient stability and primary care issues, observed ingestion of a dose, and dispensed $10 \mathrm{mg}$ methadone tablets in multidose containers. Pharmacists also supervised monthly urine toxicology testing, observing patients entering and leaving bathroom facilities and temperature testing each sample. To limit methadone diversion, patients were required to attend random medication "call-backs," returning within 24 hours for verification of appropriate methadone use and for unscheduled urine testing. Concerns regarding clinical instability triggered collaborative assessment by physicians, pharmacists, counselors, and clinical support staff to determine the need for additional monitoring and treatment. Physician visits were initially scheduled monthly; frequency was later adjusted based on clinical need. All patients were offered optional primary care at HMC and drug counseling at ETS. Physicians and pharmacists documented methadone visits in a separate methadone chart, and physicians documented nonmethadone-related medical care in the HMC medical record.

\section{Program Evaluation Methods}

Treatment status and urinalysis results were assessed through review of HMC methadone charts. Trained research staff not involved in clinical care interviewed patients at baseline, 6 months, and 12 months using the Addiction Severity Index (ASI), 5th Edition, ${ }^{16}$ and the Medical Outcomes Study Short-Form 36 (SF-36). ${ }^{17}$ Patient satisfaction was measured using standard questionnaires modified for this study ${ }^{18}$ and semistructured questions eliciting the program's effect on their lives. Tobacco use and cessation efforts, and hepatitis testing and treatment, were assessed with structured questions. A physician (JOM) reviewed each HMC medical record to assess medical diagnoses and treatments received.

Prior to training, physicians were surveyed regarding their perceived knowledge of methadone maintenance and their interest in providing methadone treatment. At 6 and 12 months, physician satisfaction was measured using standard questionnaires modified for this study. ${ }^{18}$ Prior to training and again after 6 months of practice, physician attitudes toward methadone maintenance treatment were measured using standardized questions developed originally for the evaluation of methadone clinic staff attitudes. ${ }^{19-21}$ Finally, semistructured interviews were conducted with physicians to evaluate their clinical support needs and to identify strengths and weaknesses of the program.

The University of Washington Human Subjects Committee approved all study procedures, including written informed consent from patients and physicians.

\section{Analysis}

Baseline characteristics, treatment retention, and urine drug toxicology are reported descriptively. To determine whether ASI and SF-36 scores changed over time, the scores at baseline, 6 months, and 12 months were analyzed using a randomeffects model. Specifically, we fit a linear regression model that included random participant-to-participant variability in the slope and intercept; the model accounts for correlation among repeated measures from a participant.

Patient and physician satisfaction data are reported descriptively, and data from semistructured interviews are summarized based on qualitative analysis of responses. Change in 
physician responses to questions concerning their attitudes toward methadone treatment was assessed for each individual question and for a summary score using the Wilcoxon signed ranks test.

\section{RESULTS}

\section{Regulatory Approvals}

The policy process required sequential regulatory approval, first from King County, then from the Washington State Division of Alcohol and Substance Abuse. After state approval, the FDA accepted program exemptions contingent upon approval by the Drug Enforcement Administration (DEA). The DEA process required review of program procedures at the national and regional levels, and inspection of the HMC site. The approval process began in September 1998 and ended in December 1999 with the granting of a DEA Narcotic Treatment Program license. Soon after, pharmacy protocols were approved by the Washington State Board of Pharmacy. In March 2000, the FDA and the Center for Substance Abuse Treatment (CSAT) announced the availability of methadone medical maintenance exemptions, citing our program as a model. ${ }^{22}$

\section{Eligibility and Recruitment}

Figure 1 outlines the screening process for the 684 ETS patients. Of the 109 patients with take-home privileges, 49 met our inclusion criteria and 30 agreed to enroll. If patients with 6 rather than 12 months of stability had been eligible, 3 additional patients would have qualified (data not shown). One patient who did not qualify due to outstanding fees at ETS was mistakenly permitted to enter the program, was soon found to be clinically unstable, and was transferred back to ETS.

\section{Addiction Treatment Outcomes}

Baseline patient characteristics are presented in Table 1. Although our eligibility criteria permitted entry of patients with only 1 year of clinical stability, most exhibited long-term treatment. Twenty-eight of 30 patients (93\%) remained at HMC over

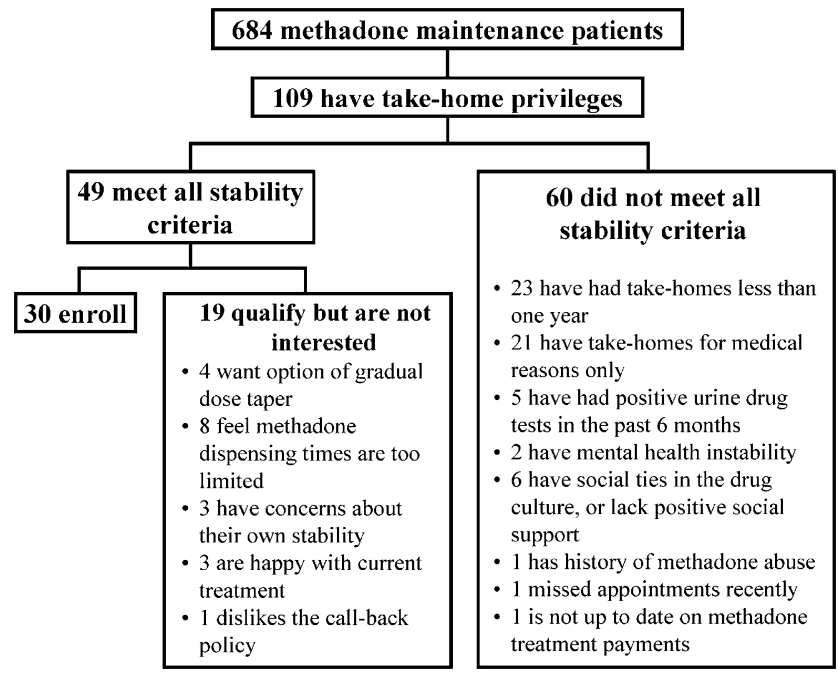

FIGURE 1. Eligibility and recruitment for methadone medical maintenance.
1 year. One transferred to a new ETS methadone program near the patient's home and a second moved from Washington State, transferring to another methadone maintenance program.

During the first year, 28 of 30 patients (93.3\%) had all negative urinalysis results (445 of 449 total tests, 99.1\%). Patients with positive urinalysis or other signs of possible instability (e.g., job stress, mental health issues) were managed with increased frequency of physician and pharmacist visits, and counseling at ETS. Overall, 19 patients had no ETS visits, five had 1 to 5 visits, three had 6 to 10 visits, and three had over 10 visits.

Random call backs $(n=33)$ involved all patients between months 4 and 12, and all call backs verified accurate methadone adherence. All associated unscheduled urinalysis tests (7.3\% of all tests) were negative for drugs of abuse and positive for methadone.

The mean ASI composite scores and SF-36 scale scores are presented in Table 2 . ASI composite scores were generally low at baseline. With the exception of the ASI medical composite score, the slopes of ASI and SF-36 scores were not significantly different from zero, suggesting that the scores did not change over time. The ASI medical composite score showed some improvement (slope $=-.014 /$ month; $P=.02$, unadjusted for multiple comparisons).

\section{Provision of Primary Medical Care}

Of the 28 patients who remained at HMC for the first year, 26 received primary care at HMC, and 2 chose only opioid dependence treatment. Visits to pharmacists (mean 26 visits, range 17 to 64 ) and physicians (mean 11 visits, range 5 to 22) allowed frequent opportunities to address primary care medical issues.

Hepatitis C Virus. By the end of the first year, 20 of 28 patients (71\%) were identified as having been exposed to hepatitis $\mathrm{C}$ virus (HCV), including 5 whose HCV status was newly clarified at HMC. Nine patients were referred for further hepatitis evaluation during the first year, 7 were seen by a hepatologist, and 1 completed HCV treatment.

Table 1. Characteristics of Methadone Medical Maintenance Patients $(N=30)$ Characteristic

\begin{tabular}{lr}
\hline \hline Mean age, y (SD) & $45(8.2)$ \\
Males, $n$ (\%) & $21(70)$ \\
White, $n$ (\%) & $25(83)$ \\
Employed/student, $n$ (\%) & $25(83)$ \\
Married/partnered, $n$ (\%) & $16(53)$ \\
Education, mean years (SD) & $14(2.4)$ \\
Age first used opioids, mean (SD) & $21(5.3)$ \\
Age first methadone treatment, mean (SD) & $30(6.9)$ \\
Methadone admits, mean (SD) & $3(1.8)$ \\
Years on methadone, mean (SD) & $12(7.8)$ \\
Current treatment duration, mean years (SD) & $10(6.9)$ \\
Years on methadone, range & $2-22$ \\
Years with take-home privileges, mean years (SD) & $7(6.8)$ \\
mg/day methadone, mean (SD) & $63(31.2)$ \\
mg/day methadone, range & $10-140$ \\
Insurance status, $n$ (\%) & \\
$\quad$ Medicaid & $9(30)$ \\
Other medical insurance & $15(50)$ \\
No insurance & $6(20)$ \\
\hline
\end{tabular}

SD, standard deviation. 
Table 2. Mean Baseline and Follow-up Addiction Severity Index* Composite Scores and SF- $36^{\dagger}$ Scale Scores for Medical Maintenance Patients $(N=28)$

\begin{tabular}{lcllr}
\hline \hline & Baseline 6-Month 12-Month & \multicolumn{1}{c}{ Slope (SE) } \\
\hline ASI scores & & & & \\
$\quad$ Medical composite & & & & \\
Employment composite & 0.34 & 0.3 & 0.17 & $-0.014(0.005)^{\S}$ \\
Alcohol composite & 0.01 & 0.32 & 0.26 & $0.000(0.003)$ \\
$\quad \begin{array}{l}\text { Drug composite } \\
\text { Legal composite }\end{array}$ & 0.09 & 0.09 & 0 & $-0.001(0.001)$ \\
Family-social composite & 0.01 & 0.02 & 0 & $-0.000(0.001)$ \\
Psychiatric composite & 0.12 & 0.07 & 0.05 & $-0.002(0.001)$ \\
$\quad$ SF-36 scores & & & 0.08 & $-0.003(0.003)$ \\
Physical functioning & 82.32 & 85.00 & 84.29 & $0.164(0.250)$ \\
$\quad$ Role limitation- & 67.86 & 74.11 & 70.54 & $0.223(0.663)$ \\
$\quad$ physical & & & & \\
$\quad$ Role limitation- & 80.95 & 82.14 & 82.14 & $0.099(0.614)$ \\
$\quad$ emotional & & & & \\
$\quad \begin{array}{l}\text { Social functioning } \\
\text { Mental health }\end{array}$ & 78.57 & 82.94 & 84.52 & $0.496(0.376)$ \\
$\quad$ Energy/vitality & 71.14 & 68.86 & 72.57 & $0.119(0.226)$ \\
$\quad \begin{array}{l}\text { Pain } \\
\text { General health }\end{array}$ & 46.61 & 52.14 & 47.68 & $0.089(0.337)$ \\
$\quad$ perception & 63.21 & 65.00 & 63.57 & $0.030(0.324)$ \\
\hline
\end{tabular}

*Addiction Severity Index (ASI) composite scores range from 0 to 1, with higher scores indicating more severe problems.

${ }^{\dagger}$ Medical Outcomes Study Short-Form 36 (SF-36) scores range from 0 to 100 with higher values indicating higher functional status.

${ }^{\ddagger}$ The ASI Medical Composite score is derived from 3 questions: 1) How many days have you experienced medical problems in the last 30 days? 2) How bothered or troubled have you been by these medical problems in the past 30 days? and 3) How important to you now is treatment for these medical problems?

${ }^{\S} \mathrm{P}=.02$.

SE, standard error; ASI, Addiction Severity Index; SF-36, Medical Outcomes Study Short-Form 36.

Tobacco Use. Seventeen patients (60.7\%) were current smokers at baseline; 14 received cessation counseling by their physicians, 8 were referred to a pharmacy-based tobacco cessation program, and 4 received pharmacotherapy for tobacco cessation. Six patients reported quit attempts during the first year, and 3 were not smoking at 12 months; 8 reported smoking less at 12 months than at baseline.

Hypertension. Eleven patients (39\%) received pharmacotherapy for hypertension. Four were treated for the first time at HMC, and 2 others changed previously established hypertension regimens.

Psychiatric Disorders. Depression and/or anxiety disorders were diagnosed and treated for the first time in 4 patients. Thirteen patients had previous psychiatric disorders, 6 of whom received changes in their pharmacotherapy during the first year.

\section{Patient Satisfaction}

Twenty-six of $30(86.7 \%)$ patients reported being very satisfied with the treatment they received and $2(6.7 \%)$ somewhat satisfied; the 2 transferred patients were not assessed. In semistructured interviews, patients expressed particular satisfaction with the reduced frequency of visits, the individualized professional care they received from physicians and pharmacists, the attention to neglected health problems, and the freedom from stigma associated with traditional OTPs. Appointment scheduling was the most frequent complaint, as it proved difficult to coordinate pharmacy and physician ap- pointments consistently. All 28 patients preferred the medical setting to a drug treatment setting, and all planned to stay in the program.

\section{Physician Assessments}

All physicians were general internists and had, on average, 10 years clinical experience after residency. None initially considered their level of knowledge sufficient to administer methadone, but all expressed interest in being trained to provide methadone to stabilized patients.

Physicians reviewed each case with a clinical support provider at least 3 times during the year. Additional clinical support requests (multiple discussions for 5 patients, occasional discussion for 6 , and little or no discussion for 18) usually concerned dose change assessment, acute medical or mental health problems, or possible patient instability. When asked after 1 year to cite areas where additional training would have been helpful, physicians most frequently mentioned the management of mental health diagnoses in the setting of addiction treatment.

Of the 10 physicians who completed the first year, 6 were completely satisfied with the educational value of the program, and 4 somewhat satisfied. In assessing satisfaction with treating each patient, physicians were completely satisfied in 14 of 28 cases $(50 \%)$, somewhat satisfied in $12(43 \%)$, and neutral or somewhat dissatisfied in $2(7 \%)$. Physicians reported very good to excellent rapport with $23(82 \%)$ patients and fair to good rapport with the rest. Comparing them to other patients in their public hospital practices, physicians generally viewed the methadone medical maintenance patients as equally or more compliant, equally or less in need of emotional support, and the same or lower on acuity of psychosocial stressors. Physicians were gratified to witness how patients benefited from the program, and all indicated willingness to care for additional methadone medical maintenance patients.

Physicians expressed concern regarding federal confidentiality rules requiring segregation of methadone-related documentation from primary care records. In providing both methadone and primary care, these physicians had to assess whether symptoms represented addiction-related instability, methadone dosing, or other medical or psychiatric disorders, and they found segregated record keeping to be artificial and constraining.

Physician attitudes toward methadone treatment became more positive after training and 6 months of methadone medical maintenance practice (Table 3). Significant changes suggesting more positive attitudes toward methadone maintenance treatment were found for 3 of 5 questions and for a summary score combining all questions. After 1 year, physicians noted significant learning about addiction and change in their views of patients with addiction problems.

\section{DISCUSSION}

Our program was the first to obtain regulatory exemptions for methadone medical maintenance in a nonexperimental setting. Critical features of this model include close affiliation with a cooperative OTP, training and clinical support for generalist physicians and pharmacists, and integrated primary care medical services. These features enabled regulatory approval and good clinical outcomes, although substantial effort was required to obtain exemptions and develop office proce- 
Table 3. Physician Attitudes Toward Methadone Maintenance Before and After Methadone Medical Maintenance Training and Practice $(N=10)$

\begin{tabular}{|c|c|c|c|}
\hline Question & Baseline Mean (SD) & $\begin{array}{l}\text { Post Training and } \\
\text { Practice Mean }{ }^{\dagger} \text { (SD) }\end{array}$ & P Value* \\
\hline $\begin{array}{l}\text { 1) No limits should be set on the duration of methadone } \\
\text { maintenance. }\end{array}$ & $4.1(0.74)$ & $4.8(0.42)$ & .02 \\
\hline $\begin{array}{l}\text { 3) Abstinence from all opioids (including methadone) should be } \\
\text { the principal goal of methadone maintenance. } .^{\ddagger}\end{array}$ & $3.9(0.99)$ & $4.7(0.48)$ & .01 \\
\hline $\begin{array}{l}\text { 5) When a methadone maintenance patient becomes pregnant, } \\
\text { she should be withdrawn from methadone. }\end{array}$ & $4(0.82)$ & $4.5(0.71)$ & .06 \\
\hline Summary score & $19.8(2.86)$ & $23.1(2.51)$ & .01 \\
\hline
\end{tabular}

*P values were obtained from Wilcoxon signed rank test (two-tailed), which tests the null hypothesis that each change comes from a distribution that is symmetric with a mean of zero.

${ }^{\dagger}$ Based on a 5-point scale (strongly agree to strongly disagree) with higher scores indicating more positive attitudes toward methadone maintenance treatment.

${ }^{\ddagger}$ Before calculating means, scores were reversed for questions 2,3 , and 5 so that a higher value indicated more positive attitudes toward methadone maintenance.

SD, standard deviation.

dures and protocols. Our program applied to a minority of methadone maintenance patients, but given the close to 200,000 patients receiving methadone treatment in the United States today, extending this model could enhance care for a significant number of stable patients.

Patients remained stable after transferring to methadone medical maintenance. Our addiction outcomes were comparable to those of earlier successful experimental programs, ${ }^{9,14,15}$ and in addition we found possible improvement in patients' medical status over time. Similar temporal improvements were not found in previous office-based methadone trials that used the same ASI medical status measure. ${ }^{18,23}$ These trials, however, provided no integrated primary care services. While our observational design is limited, these results suggest that when methadone medical maintenance delivers multiple interventions for previously unaddressed medical problems, important health gains result. The benefits of medical care in conjunction with addiction treatment have been documented in other settings ${ }^{24-27}$ and add to the rationale for integrating these systems of care.

Affiliation with an existing OTP was required for regulatory approval and guarantees continuity of care if patients relapse and need more frequent methadone dispensing. ETS fully supported providing these services to eligible patients. Other programs might be concerned that the transfer of stable patients could affect staff morale or put a significant strain on revenues, as many programs receive comparable reimbursement for the most stable patients and for new patients with multiple problems. This could impede widespread implementation of methadone medical maintenance. However, patients who can benefit from a lower intensity of care should not suffer from such adverse payment mechanisms.

Our program is unique in providing methadone maintenance treatment in the "real world" medical practices of generalist physicians with no previous addiction medicine experience. Our brief initial training program and ongoing clinical support and consultation parallels practice patterns in other medical specialties. Physicians expressed satisfaction with this approach, and although we studied a small number of physicians, there was evidence of increasingly positive attitudes toward methadone maintenance. Such attitudes have been associated with improved patient treatment retention. ${ }^{28}$ Physicians perceived co-occurring mental health issues as a challenging aspect of care for this population, making this a potential target for additional training.

No previous U.S. methadone medical maintenance program has incorporated clinical pharmacists into methadone dispensing and assessment of patients. Regular pharmacist contact with patients facilitated their major role in clinical care coordination. Pharmacists have been involved in the expansion of methadone treatment capacity in other countries, ${ }^{29}$ and could play a similar role in the United States.

Our small sample size did not allow for meaningful cost estimates, though costs will influence widespread implementation of this model. Reduced addiction services utilization has been documented in methadone medical maintenance due to additional take-home methadone doses. ${ }^{14}$ However, Washington State methadone maintenance reimbursement policy does not allow a commensurate reduction in charges for patients in methadone medical maintenance. Thus, the additional provision of primary medical care in conjunction with addiction treatment monitoring may have increased overall costs of care while adding the benefits of medical services. Initial grant funding was required to develop and evaluate this program but did not support clinical services, allowing the continuation of this program as a self-funding, collaborative project of HMC and ETS.

The relatively infrequent monitoring of patients in methadone medical maintenance may have missed some drug use, as more frequent testing and hair analysis has detected more use in a similar population. ${ }^{18}$ However, unremitting dependent use is likely to be detected by monthly tests and clinical observations, and the clinical significance of intermittent use in this setting is not clear. Random call backs discovered no additional drug use or major irregularities in methadone adherence, and these have been continued for patients remaining in our program. This diversion control measure was not overly burdensome and may encourage patient adherence while providing verification of appropriate methadone use.

Methadone medical maintenance cannot substantially address the urgent need to increase access to initial methadone treatment in the United States, particularly for those in need of public funding. Other countries have successfully in- 
creased access to methadone by allowing generalist physicians to initiate treatment, with outcomes comparable to clinicbased practice. ${ }^{29-32}$ In the United States, initial treatment may be provided by trained and certified physicians using buprenorphine, a new medication recently approved by the FDA for treatment of opioid dependence. ${ }^{33}$ The reduced regulatory burden that applies to buprenorphine compared with methadone medical maintenance may make it more attractive to physicians. However, access to buprenorphine or methadone will remain restricted unless funding levels for addiction treatment are increased or parity between medical and addiction treatment insurance is established.

Recent changes in federal regulations permit OTPs new clinical flexibility. ${ }^{34}$ Clinics may now give 1-month supplies of take-home methadone to successful patients after 2 years in treatment, addressing part of the rationale for methadone medical maintenance. However, integrated methadone medical maintenance can provide the additional benefits of enhancing patient satisfaction with methadone treatment, reducing patient contact with less stabilized patients, developing physician expertise in addictions, and improving the medical care of patients in methadone treatment. The new regulations explicitly request exemption applications to create methadone medical maintenance programs and thus expand access to this care model for other stabilized methadone maintenance patients.

While complex, it is feasible to obtain regulatory approval and train generalist physicians and pharmacists to provide methadone medical maintenance with good patient outcomes, high levels of patient and provider satisfaction, and potential improvement in physician attitudes toward methadone treatment. While the complexity of regulatory policy and program protocol development is substantial and may limit expansion of this model of care, the precedent set by our program should facilitate smoother regulatory approval for future programs.

This work was assisted by a grant from the Robert Wood Johnson Foundation Substance Abuse Policy Research Program (RWJF grant 34895). We are grateful for the data management and statistical support of Katie Weaver, for the comments of Elizabeth Dickinson on earlier versions of the manuscript, and for the participation of patients and providers.

\section{REFERENCES}

1. Dole VP. Implications of methadone maintenance for theories of narcotic addiction. JAMA. 1988;260:3025-9.

2. Effective medical treatment of opiate addiction. National Consensus Development Panel on Effective Medical Treatment of Opiate Addiction. JAMA. 1998;280:1936-43.

3. Barnett PG, Hui sS. The cost-effectiveness of methadone maintenance. Mt Sinai J Med. 2000;67:365-74.

4. Ball J. The Effectiveness of Methadone Maintenance Treatment: Patients, Programs, Services, and Outcomes. New York, NY: Springer-Verlag; 1991.

5. Sees KL, Delucchi KL, Masson C, et al. Methadone maintenance vs 180-day psychosocially enriched detoxification for treatment of opioid dependence: a randomized controlled trial. JAMA. 2000;283:1303-10.

6. Gossop M, Marsden J, Stewart D, Treacy S. Outcomes after methadone maintenance and methadone reduction treatments: two-year follow-up results from the National Treatment Outcome Research Study. Drug Alcohol Depend. 2001;62:255-64.

7. Rettig RA, Yarmolinsky A, eds. Federal Regulation of Methadone Treatment. Washington, DC: National Academy Press; 1995.

8. Merrill JO. Policy progress for physician treatment of opiate addiction. J Gen Intern Med. 2002;17:361-8.
9. Novick DM, Pascarelli EF, Joseph H, et al. Methadone maintenance patients in general medical practice. A preliminary report. JAMA. 1988;259:3299-302.

10. Novick DM, Joseph H. Medical maintenance: the treatment of chronic opiate dependence in general medical practice. J Subst Abuse Treat. 1991;8:233-9.

11. Novick DM, Richman BL, Friedman JM, et al. The medical status of methadone maintenance patients in treatment for 11-18 years. Drug Alcohol Depend. 1993;33:235-45.

12. Salsitz EA, Joseph H, Frank B, et al. Methadone medical maintenance (MMM): treating chronic opioid dependence in private medical practicea summary report (1983-1998). Mt Sinai J Med. 2000;67:388-97.

13. Schwartz RP, Brooner RK, Montoya ID, Currens M, Hayes M. A 12 year follow-up of a methadone medical maintenance program. Am J Addict. 1999;8:293-9.

14. King VL, Stoller KB, Hayes M, et al. A multicenter randomized evaluation of methadone medical maintenance. Drug Alcohol Depend. 2002;65: 137-48.

15. Senay EC, Barthwell A, Marks R, Bokos PJ. Medical maintenance: an interim report. J Addict Dis. 1994;13:65-9.

16. McLellan AT, Kushner H, Metzger D, et al. The Fifth Edition of the Addiction Severity Index. J Subst Abuse Treat. 1992;9:199-213.

17. Ware JE Jr, Sherbourne CD. The MOS 36-item short-form health survey (SF-36). I. Conceptual framework and item selection. Med Care. 1992;30:473-83.

18. Fiellin DA, O'Connor PG, Chawarski M, Pakes JP, Pantalon MV, Schottenfeld RS. Methadone maintenance in primary care: a randomized controlled trial. JAMA. 2001;286:1724-31.

19. Caplehorn JR, Irwig L, Saunders JB. Attitudes and beliefs of staff working in methadone maintenance clinics. Subst Use Misuse. 1996;31:437-52

20. Caplehorn JR, Hartel DM, Irwig L. Measuring and comparing the attitudes and beliefs of staff working in New York methadone maintenance clinics. Subst Use Misuse. 1997;32:399-413.

21. McNeely J, Drucker E, Hartel D, Tuchman E. Office-based methadone prescribing: acceptance by inner-city practitioners in New York. J Urban Health. 2000;77:96-102.

22. Lepay D, Clark Hw. Federal Advisory on Methadone Medical Maintenance Exemptions. Washington, DC: Department of Health and Human Services; 2000

23. Senay EC, Barthwell AG, Marks R, Bokos P, Gillman D, White R. Medical maintenance: a pilot study. J Addict Dis. 1993;12:59-76.

24. Weisner C, Mertens J, Parthasarathy S, Moore C, Lu Y. Integrating primary medical care with addiction treatment: a randomized controlled trial. JAMA. 2001;286:1715-23.

25. Laine C, Hauck WW, Gourevitch MN, Rothman J, Cohen A, Turner BJ. Regular outpatient medical and drug abuse care and subsequent hospitalization of persons who use illicit drugs. JAMA. 2001;285:235562.

26. Willenbring ML, Olson DH. A randomized trial of integrated outpatient treatment for medically ill alcoholic men. Arch Intern Med. 1999; 159:1946-52.

27. Friedmann PD, Zhang Z, Hendrickson J, Stein MD, Gerstein DR. Effect of primary medical care on addiction and medical severity in substance abuse treatment programs. J Gen Intern Med. 2003;18:1-8.

28. Caplehorn JR, Lumley TS, Irwig L. Staff attitudes and retention of patients in methadone maintenance programs. Drug Alcohol Depend. 1998;52:57-61.

29. Weinrich M, Stuart M. Provision of methadone treatment in primary care medical practices: review of the Scottish experience and implications for US policy. JAMA. 2000;283:1343-8.

30. Hutchinson SJ, Taylor A, Gruer L, et al. One-year follow-up of opiate injectors treated with oral methadone in a GP-centred programme. Addiction. 2000;95:1055-68.

31. Gossop M, Stewart D, Browne N, Marsden J. Methadone treatment for opiate dependent patients in general practice and specialist clinic settings: outcomes at 2-year follow-up. J Subst Abuse Treat. 2003;24:313-21.

32. Brands J, Brands B, Marsh D. The expansion of methadone prescribing in Ontario, 1996-1998. Addict Res. 2000;8:485-96.

33. Fiellin DA, O'Connor PG. Clinical practice. Office-based treatment of opioid-dependent patients. N Engl J Med. 2002;347:817-23.

34. Opioid drugs in maintenance and detoxification treatment of opiate addiction; Substance Abuse and Mental Health Services Administration, Department of Health and Human Services. Final rule. Fed Regist. 2001;66:4076-102. 